\title{
Different Response Profiles of Gastrointestinal Cancer Cells to an L-Type Amino Acid Transporter Inhibitor, JPH203
}

\author{
YASUHIDE MUTO ${ }^{1,2}$, TOMOMI FURIHATA ${ }^{2}$, MEIKA KANEKO ${ }^{2}$, KOSUKE HIGUCHI $^{2}$, KENTARO OKUNUSHI $^{2}$, \\ HANAE MORIO $^{2}$, YOSHIE REIEN ${ }^{2}$, MASAYA UESATO $^{1}$, HISAHIRO MATSUBARA $^{1}$ and NAOHIKO ANZAI ${ }^{2,3}$ \\ ${ }^{1}$ Department of Frontier Surgery, Chiba University Graduate School of Medicine, Chiba, Japan; \\ ${ }^{2}$ Department of Pharmacology, Chiba University Graduate School of Medicine, Chiba, Japan; \\ ${ }^{3}$ Department of Pharmacology and Toxicology, Dokkyo Medical University School of Medicine, Tochigi, Japan
}

\begin{abstract}
Background/Aim: L-type amino acid transporter 1 (LAT1) is a promising molecular target for cancer therapy. The present study aimed to characterize the anti-cancer effects of JPH203, an LAT1-selective inhibitor, on gastrointestinal cancer cells. Materials and Methods: Three esophageal, two gastric, and two colon cancer cell lines were used. Cytotoxic effects of JPH2O3 were assessed by a WST-8 assay. LAT1 mRNA levels were determined by quantitative PCR. The inhibitory property of JPH2O3 against LAT1 function was examined by a transport assay. Results: JPH2O3 treatment significantly reduced the viability of all gastric and colon cancer cells. While LAT1 expression levels and inhibitory potencies of JPH2O3 on LATI functions were comparable among the cells, all the esophageal cells were resistant to JPH2O3. Conclusion: JPH2O3 was effective in reducing gastric and colon cancer cells. To clarify its cell type-dependent efficacy, identification of the causal factors for JPH2O3 resistance will be needed.
\end{abstract}

Amino acids serve as essential components of cellular proteins as well as important energy sources, and thus they are indispensable for cell proliferation. Among the known amino acid transport systems, the system $\mathrm{L}$ is responsible for a $\mathrm{Na}^{+}$-independent neutral amino acid uptake pathway, including several essential amino acids such as leucine (1$3)$. The system L consists of four members: L-type amino acid transporter 1 (LAT1, SLC7A5), LAT2 (SLC7A8), LAT3 (SLC43A1), and LAT4 (SLC43A2), with LAT1 and LAT2 requiring heterodimerization with $\mathrm{CD} 98$ to fulfill their transporter functions.

Correspondence to: Naohiko Anzai, MD, Ph.D, Department of Pharmacology, Chiba University Graduate School of Medicine, 18-1 Inohana, Chuo-ku, Chiba 260-8670, Japan. Tel/Fax: +81 432262050/2052,e-mail: anzai@chiba-u.jp

Key Words: L-type amino acid transporter 1, JPH203, gastric cancer, colon cancer, anti-cancer drug.
Experimental and clinical evidence have shown that LAT1 becomes overexpressed during the course of development of various solid cancers, including prostate, breast, lung, pancreatic, and gastrointestinal cancers (4-7). In these cancers, it has been shown that strong LAT1 immunostaining is exclusively observed in cancerous tissues, and LAT1 overexpression, thus, appears to associate with cancer (8). For patients with esophageal cancer, it has been reported that patients with high LAT1 expression levels have significantly poorer overall survival and recurrence-free survival rates than patients with low expression levels (9). Similar results can be found for patients with non-scirrhous gastric cancer, in which high LAT1 expression levels are associated with a lower overall survival rate (10). While such an association has yet to be confirmed in colorectal cancer, a recent study showed that LAT 1 expression levels are higher in cases with a higher degree of invasion depth and venous invasion (11).

Based on its cancer-associated expression profiles together with the fact that LAT1 provides a supply route for essential amino acids required for cell proliferation, LAT1 has been acknowledged as a promising molecular target for cancer therapy. Accordingly, JPH203 (or KYT-0353), which is a tyrosine analog, has been developed as a novel anti-cancer agent specifically inhibiting LAT1 function $(12,13)$. It has been presumed that JPH203 shuts down LAT1-mediated amino acid uptake in cancer cells, resulting in a nutrient starvation status that will cause retardation of cell proliferation and eventually cell death. In accordance with this, anti-cancer effects of JPH203 have been demonstrated in pre-clinical experiments using several cancer cell types, such as cholangiocarcinoma (14), oral cancer (15), osteosarcoma (16), and thymic carcinoma cells (17). Furthermore, a first-inhuman phase I study examining the effects of JPH203 in 17 patients with advanced solid tumors has recently been launched, the ongoing results of which show that JPH203 is well-tolerated and effective against biliary tract cancer (18).

Given the results obtained so far, it can be expected that JPH203 is an emerging anti-cancer drug that effectively 
fights against various solid cancer types. Nevertheless, due to its short history, experimental evidence regarding the pharmacological characterization of JPH203 is limited, and thus its efficacy profile in several cancer types, including gastrointestinal cancers (esophageal, gastric, and colon cancers), remains to be elucidated.

Therefore, in order to expand our knowledge regarding the pharmacological characteristics of JPH203, this study aimed at clarifying its anti-cancer effects on esophageal, gastric, and colon cancers using several cell lines that have not been tested so far.

\section{Materials and Methods}

Cell lines and culture. Human esophageal cancer cell lines (TE5, TE6, and TE14) were kindly provided by Dr. Nishihira (Tohoku University, Sendai, Japan). Human gastric adenocarcinoma cell lines (MKN1 and MKN45) and a human colon adenocarcinoma cell line (Lovo) were obtained from Health Science Research Resources Bank (Osaka, Japan), and another human colon adenocarcinoma cell line (HT29) was obtained from American Type Culture Collection (Manassas, VA, USA).

TE cells were cultured in Dulbecco's Modified Eagle's medium (DMEM)/Nutrient Mixture F-12 Ham (Sigma-Aldrich, St Louis, MO, USA). MKN1 and MKN45 cells were cultured in RPMI 1640 Medium (Nacalai Tesque, Kyoto, Japan). Lovo and HT29 cells were cultured in Ham F-12 Medium (Sigma-Aldrich) and McCoy's 5a Medium Modified (Thermo Fisher Scientific, Waltham, MA, USA), respectively. All culture media were supplemented with $10 \%$ fetal bovine serum and penicillin-streptomycin. All cells were cultured in a humidified incubator at $37^{\circ} \mathrm{C}$ with $5 \% \mathrm{CO}_{2}$.

Cell viability assay. Inhibitory effects of JPH203 on cell proliferation activity were evaluated by a cell viability test using the WST-8 kit (Nacalai Tesque) according to the manufacturer's protocol. Cells were seeded in 96-well plates at a density that had been determined by preliminary experiments and were cultured for $24 \mathrm{~h}$. The cells were exposed to JPH203 (0.01, 0.1, 1, 3, 10, 30, $50 \mu \mathrm{M})($ MedKoo, Morrisville, NC, USA) for $96 \mathrm{~h}$. Following treatment, cell numbers were determined. In different experiments, combinatory effects of the following compounds on JPH203 $(20 \mu \mathrm{M})$ were also examined: chloroquine (an autophagy inhibitor, $30 \mu \mathrm{M})$ (Wako, Osaka, Japan), SP600125 (a c-Jun N-terminal kinase [JNK] inhibitor, $30 \mu \mathrm{M}$ ) (Wako), SL327 (a mitogen-activated protein kinase kinase [MEK] inhibitor, $5 \mu \mathrm{M}$ ) (Wako), parthenolide (a nuclear factor-kB [NF-kB] inhibitor, $5 \mu \mathrm{M}$ ) (LKT Laboratories, St. Paul, MN), and SB202190 (a p38 inhibitor, $20 \mu \mathrm{M}$ ) (Wako), or AKT inhibitor VIII (an Akt inhibitor, $10 \mu \mathrm{M}$ ) (Tokyo Chemical Industry, Tokyo, Japan). Dimethyl sulfoxide (DMSO, which is the solvent of the chemicals) was used as a control and set at the final concentration of $0.5 \%$ throughout the experiments

$\mathrm{IC}_{50}$, which is the concentration at which the cell viability is reduced by half, was calculated using GraphPad Prism 7J (GraphPad Software, La Jolla, CA, USA).

TUNEL assay. TE14, MKN1 and Lovo cells were seeded as described above, and were exposed to $30 \mu \mathrm{M}$ JPH203 for $72 \mathrm{~h}$. Apoptosis of treated cells was detected using TUNEL using the in situ apoptosis detection kit (Takara-bio, Shiga, Japan) according to the manufacturer's protocol.
Table I. Primers.

\begin{tabular}{lll}
\hline mRNA & & Primer $\left(5^{\prime}>3^{\prime}\right)$ \\
\hline LAT1 & Forward & TGTACGTGCTGACCAACCTG \\
& Reverse & ATGACGCCCAGGTGATAGTTC \\
LAT2 & Forward & CACGGTTGCTGGACAGATAG \\
& Reverse & GGGAACAGCAGGTTGATCTT \\
LAT3 & Forward & ATGGACTGGCGGATCAAGG \\
& Reverse & TCTTGCAGTAGCGTGGTCTGATG \\
LAT4 & Forward & GGTGGACAAGTTTCTGCTGAGTG \\
& Reverse & CAGCTGTATGAGGATGCGGTGTA \\
$\beta$-actin & Forward & CCAACCGCGAGAAGATGA \\
& Reverse & CCAGAGGCGTACAGGGATAG \\
\hline
\end{tabular}

Quantitative PCR ( $q P C R$ ). LAT1, LAT2, LAT3, and LAT4 mRNA expression levels in gastrointestinal cancer cells were quantified by qPCR. Total RNA of gastrointestinal cancer cells was isolated using ISOGEN II (Nippon Gene, Tokyo, Japan). First-strand cDNA was synthesized from $1.0 \mu \mathrm{g}$ of total RNA using ReverTra Ace qPCR RT master mix with a gDNA Remover (Toyobo, Osaka, Japan).

The qPCR analysis was performed using specific primers (Table I) and Thunderbird SYBR qPCR Mix (Toyobo). The relative expression levels were determined using the $\Delta \Delta \mathrm{Ct}$-method with $\beta$ actin mRNA level as a normalization control.

Leucine transport assays. LAT1 function, along with the inhibitory effect of JPH203, in gastrointestinal cancer cells was examined by transport assays using L-leucine (leucine), which is a prototypical LAT1 substrate. Two days after gastrointestinal cancer cells had been seeded in a 24 -well plate $\left(10^{4}\right.$ cells/well $)$, the transport assay was performed. The cell culture medium was replaced with Hanks' solution and pre-incubated for $5 \mathrm{~min}$. The solution was then replaced with fresh Hanks' solution with leucine (containing $\left[{ }^{14} \mathrm{C}\right]$ leucine at $\left.0.1 \mathrm{mCi} / \mathrm{ml}\right)$ (PerkinElmer, Boston, MA) at the final concentration of $1.0 \mu \mathrm{M}$, and the cells were incubated for one min at $37^{\circ} \mathrm{C}$. In the inhibition assays, JPH203 $(10 \mu \mathrm{M})$ or excess amount of non-radiolabeled leucine $(500 \mu \mathrm{M})$ were also added to the substrate solution. After washing three times with cold Hanks' solution, the cells were solubilized with $0.1 \mathrm{~N} \mathrm{NaOH}$ and their radioactivity was determined using a $\beta$-scintillation counter (2810TR, Perkin Elmer). Uptake activity was normalized for protein content, which was determined by a Pierce BCA protein assay kit (Thermo Fisher Scientific). Finally, the background activity (which was obtained in the presence of excess cold leucine $[500 \mu \mathrm{M}]$ ) was subtracted from each uptake activity.

\section{Results}

Growth inhibition profile of human gastrointestinal cancer cells treated with JPH203. To examine the effects of JPH203 on esophagus (TE5, TE6 and TE14), gastric (MKN1 and MKN45), and colon (Lovo and HT29) cancer cell proliferation, the cells were treated with JPH203 at various concentrations for $96 \mathrm{~h}$ and then WST-8 assays were performed. As shown in Figure 1, the growth rates of MKN1, MKN45, Lovo, and HT29 cells were significantly reduced upon JPH203 treatment: 

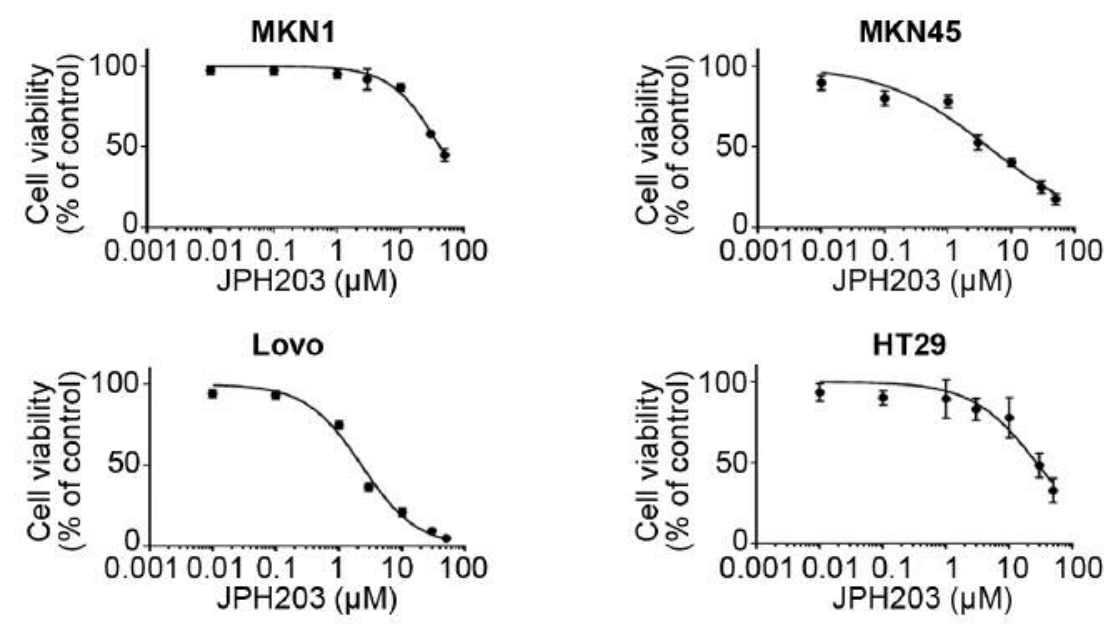

TE5

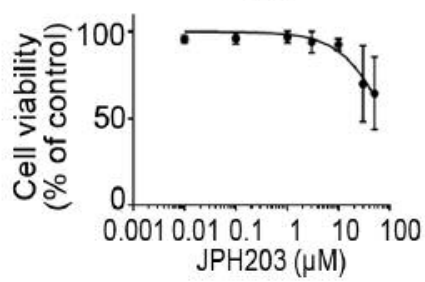

TE6

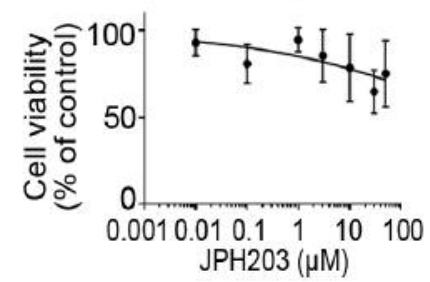

TE14

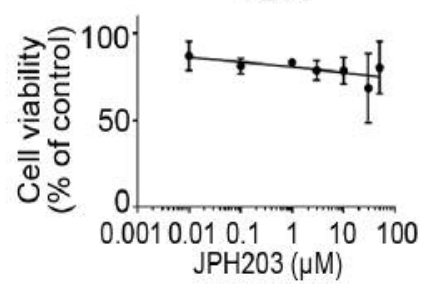

Figure 1. Effects of JPH2O3 on the viability of gastrointestinal cancer cells. Esophagus (TE5, TE6 and TE14), gastric (MKN1 and MKN45), and colon (Lovo and HT29) cancer cells were treated with JPH2O3 at various concentrations for 96 , and their viabilities were analyzed using the WST-8 assay. The viability at each concentration (\%) was calculated with respect to the control value (100\%), which was obtained from cells treated with DMSO (0.5\%). Data are expressed as means with S.D. Using these results, the $I C_{50}$ value for each cell type was calculated (Table II). The experiments were repeated three to five times, in triplicate.

For example, at $50 \mu \mathrm{M}$ of $\mathrm{JPH} 203$, the growth rates of the cancer cells were $45.0 \pm 3.9 \%, 17.8 \pm 3.5 \%, 5.0 \pm 0.7 \%$, and $33.0 \pm 3.7 \%$ of the growth rate of control cells, respectively. Therefore, the results clearly show that JPH203 exerts inhibitory effects on the growth of gastric and colon cancers.

In contrast, all TE cells were highly resistant to the treatment $(64.6 \pm 10.4 \%, 76.0 \pm 10.2 \%, 80.4 \pm 7.6 \%$, respectively) even at $50 \mu \mathrm{M}$.

The $\mathrm{IC}_{50}$ values of JPH203 in gastrointestinal cells are summarized in Table II.

Induction of apoptosis by JPH2O3 in gastrointestinal cancer cells. In order to determine whether the inhibition of cell proliferation by JPH203 resulted from its static effects or induction of cell death, TUNEL assays were performed (Figure 2). Increased staining in MKN1 and Lovo cells indicates that apoptosis is involved in JPH203-mediated cell death. As expected, TE14 cells, which were resistant to JPH203, did not show any sign of apoptosis.

Expression profile of LATs in gastrointestinal cancer cells. To compare the mRNA expression levels of LAT1, LAT2, LAT3, and LAT4 in gastrointestinal cancer cells, qPCR was performed (Figure 3). LAT1 mRNA expression was clearly observed in all the cells examined. LAT2 and LAT3 mRNA expression and, to a lesser extent, LAT4 mRNA expression were observed in some cells, but their levels were substantially lower than those of LAT1. Of note, the mRNA expression levels of these LAT members were not apparently related to the JPH203 sensitivity profile of the cells.

Leucin uptake activities and their Inhibition by JPH2O3 in gastrointestinal cancer cells. Leucine uptake activities in the presence of $\mathrm{Na}^{+}$were examined to characterize LAT1 functional levels as well as the inhibitory effect of JPH203 on LAT1 function in gastrointestinal cancer cells. As shown in Figure 4A, leucine uptake activity was clearly detected in all cancer cells regardless of their JPH203 sensitivity profiles. Furthermore, these activities were considerably suppressed in the presence of JPH203 to comparable degrees, and these suppressed fractions accounted for 70$90 \%$ of total uptake in each cell type (Figure 4B).

Thus, our results suggest that while LAT1 is responsible for most of the leucine uptake activity, no striking differences in its functional levels as well as its sensitivity profile against JPH203 were observed among esophageal, gastric and colon cancer cells. 


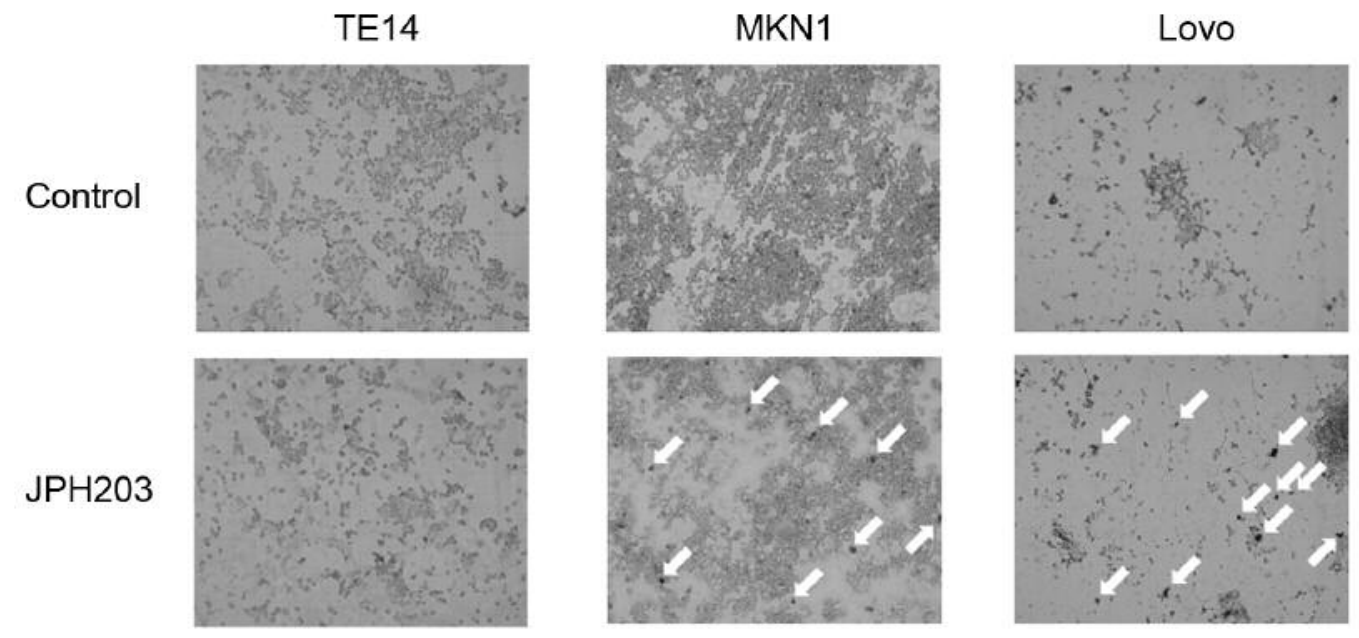

Figure 2. Detection of JPH203-induced apoptosis in gastrointestinal cancer cells. Fragmented DNA associated with JPH203-induced apoptosis was detected by TUNEL staining. JPH2O3-sensitve (MKN1 and Lovo) and -resistant (TE14) cells were incubated with JPH203 (30 $\mu M$ ) for 72 h. The cells with dark staining of nuclei (arrows) were designated as apoptotic cells. The experiments were repeated three times, and representative results are shown.

Exploration of a JPH2O3 resistance factor in esophageal cancer cells. In order to obtain insights into a causal factor for the resistance of TE5, TE6 and TE14 cells in JPH203, exploratory experiments were performed. First, considering that autophagy activity may counteract amino acid deficiency, chloroquine (an autophagy inhibitor) was added in combination with JPH203. However, it did not ameliorate JPH203 resistance (data not shown).

Next, effects of inhibitors for cell survival signaling pathways (JNK, MEK, NF-kB, p38, and AKT) on cell proliferation were examined in the presence or absence of JPH203. Unfortunately, however, the inhibitors did not show any synergistic effects with JPH203 (data not shown).

\section{Discussion}

The efficacy of JPH203 has not been examined in any cell lines used here (except for HT29 cells) and our results show for the first time that JPH203 efficiently inhibits growth rate of gastric cancer cells. Also, clear susceptibility of Lovo cells to the agent is consistent with the results of a previous study using HT29 cells (12). Therefore, these findings indicate the promising possibility that JPH203 is effective against gastric and colon cancers, which will not only encourage further JPH203 development studies but also contribute to the establishment of LAT1 inhibition as an emerging, as well as promising, concept of anti-cancer treatment.

Aside from these exciting views, a lack of (or very weak) anti-cancer effects of JPH203 on all the esophageal cancer cells examined was found. This might result from either their
Table II. $I C_{50}$ values.

\begin{tabular}{lccccccc}
\hline Cell line & TE5 & TE6 & TE14 & MKN1 & MKN45 & Lovo & HT29 \\
\hline $\mathrm{IC}_{50} \mathrm{a}(\mu \mathrm{M})$ & - & - & - & $41.7 \pm 2.3$ & $4.6 \pm 1.0$ & $2.3 \pm 0.3$ & $30.0 \pm 6.4$
\end{tabular}

aHyphens indicate that the value cannot be calculated.

nature of esophageal origin or their own intrinsic properties. While it is currently unknown which is true, this finding may provide an important insight into future JPH203 development studies. That is, it is possible that JPH203 shows a patient- or tissue-dependent activity. Since therapeutic effects of anti-cancer drugs generally vary among patients or tissues, it is not so surprising if this is the case for JPH203. Rather, the critical point is to identify how JPH203 efficacy differs and what can determine it.

Most of the studies on JPH203 have focused on its efficacy (12-17), and the causal factor for the development of resistance of certain cancer cells has thus not been identified. Nevertheless, based on the mode-of-action of JPH203, it seems reasonable to hypothesize that loss of LAT1 in TE cells or aberrant LAT1 sensitivity to JPH203 might play a role in the acquired resistance in esophageal cancer cells. However, the fact that LAT1 expression levels are comparable among the cell lines tested and the fact that JPH203 reduced leucine uptake activity (namely LAT1 function) to similar levels in all cells do not support the LAT1-related hypotheses. Therefore, $\mathrm{JPH} 203$ resistance of TE cells is unlikely to be explained by 

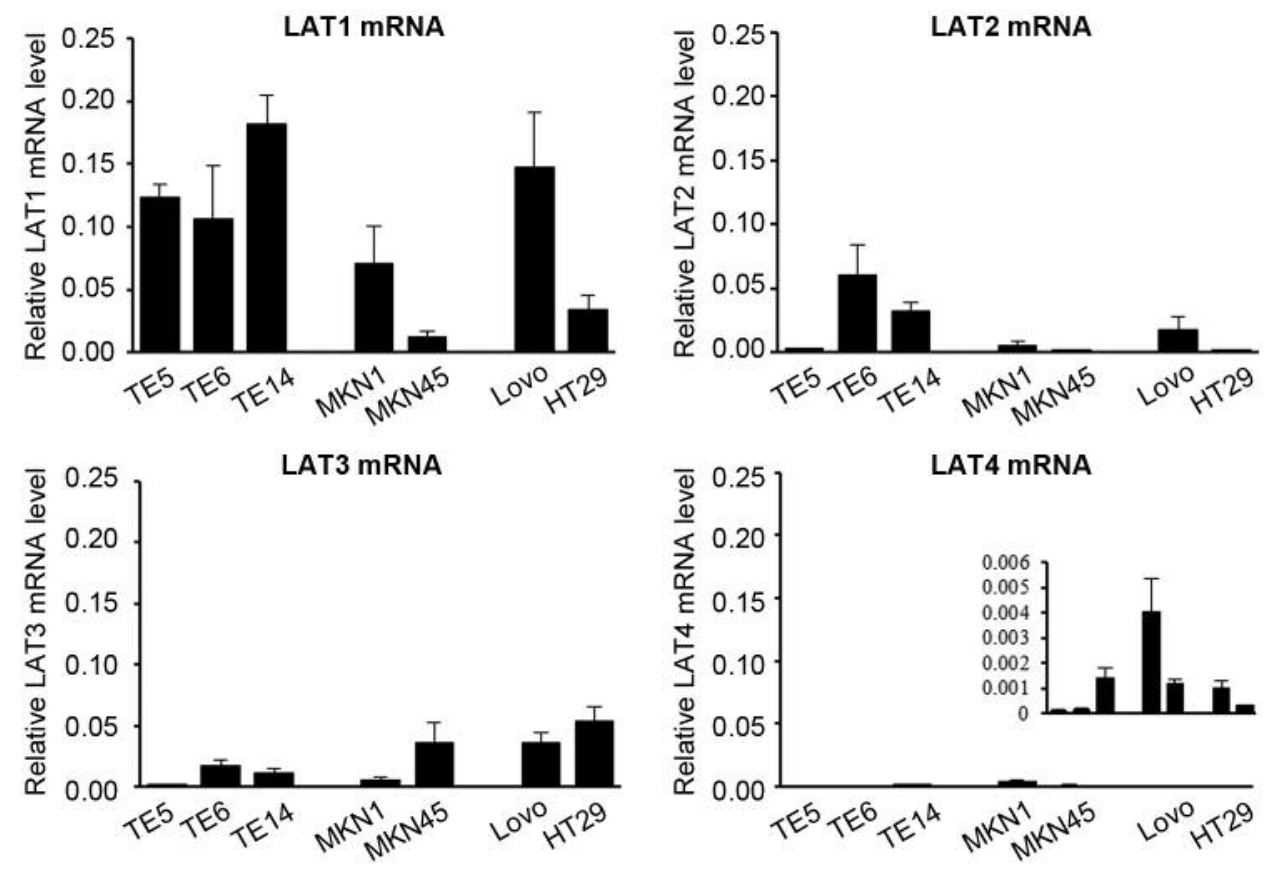

Figure 3. Quantification of mRNA expression levels of LAT family members in gastrointestinal cancer cells. qPCR was performed to determine LAT1, LAT2, LAT3, and LAT4 mRNA expression levels in each cell type. Experiments were performed at least three times, in duplicate. The expression levels were normalized to those of $\beta$-actin, and data are expressed as means with S.D.

A

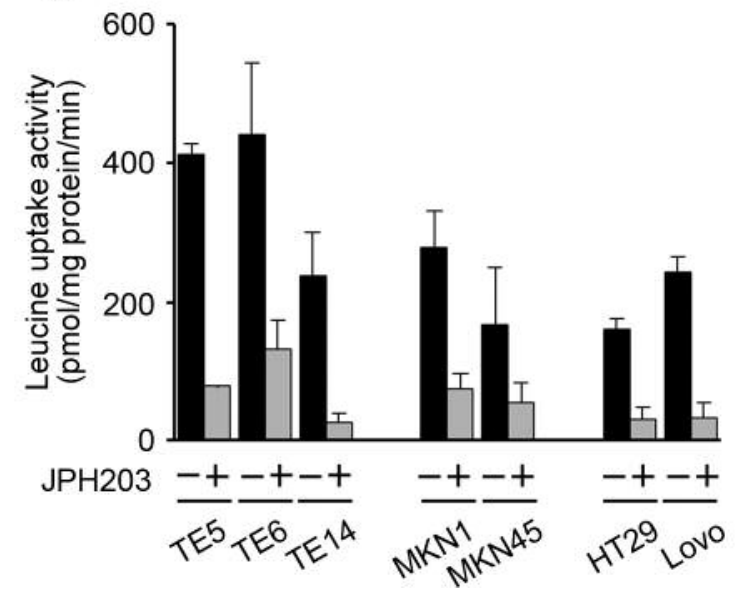

B

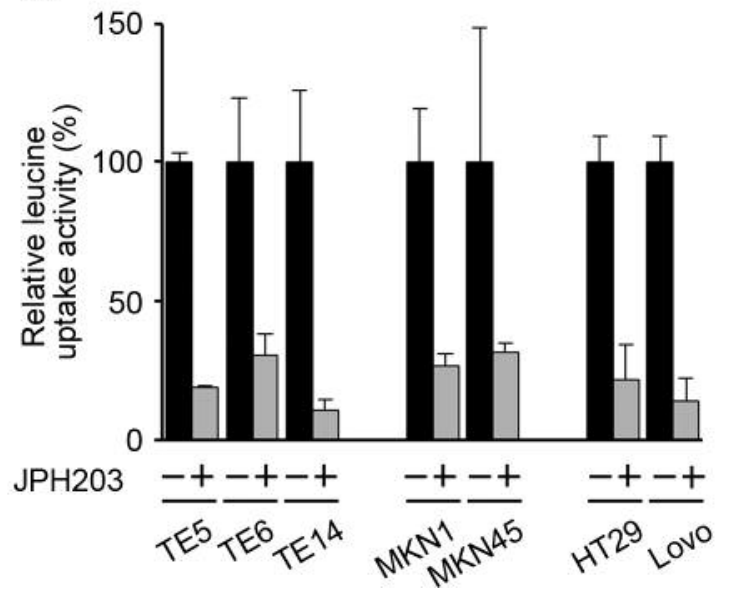

Figure 4. Determination of leucine uptake activities and their sensitivities against JPH2O3 in gastrointestinal cancer cells. Leucine uptake activities in gastrointestinal cancer cells were determined by transport assays using $\left[{ }^{14} \mathrm{C}\right]$ leucine $(1 \mu \mathrm{M})$ in the presence $(+)$ or absence $(-)$ of JPH203 $(10 \mu \mathrm{M})$. The transport assays were conducted in the presence of $\mathrm{Na}^{+}$. The gray bars indicate LAT1-independent leucine uptake activities. A and $B$ show the absolute uptake activity and relative uptake activity, respectively. In B, the values in the absence of JPH203 were set at $100 \%$. Determinations were performed three to five times, in duplicate. Each value represents the mean with S.D.

LAT1 functionality. Importantly, this point leads to the possibility that while LAT1 detection in cancer tissues warrants JPH203-based therapy, it does not always ensure its efficacy; that is, significant individual differences in the therapeutic effects in LAT1-positive patients can be expected.
On the other hand, our results also indicate that there should be another factor (or other factors) causing JPH203 resistance. In an attempt to identify the factor(s), the following two possibilities were examined: i) counterbalancing leucine deficiency with compensatory activation of its supply pathways 
and ii) enhancing cell survival systems that fight against amino acid starvation-induced cell death.

As for the first possibility, the involvement of amino acid transporters other than LAT1 in compensatory supply of essential amino acids is unlikely. This is because, although such transporter activity can be detected (i.e., JPH203resistant uptake fractions), their overall contribution to leucine uptake is low and they are not significantly different among the cells. Consistently, LAT2-4 mRNA expression, if any, is low, and there is no remarkable difference between their expression levels in TE cells and other cells. Additionally, the possibility of intracellular leucine pool manipulation through autophagy, which is a well-known process by which cells can obtain amino acids in a starved condition was examined $(19,20)$. Unfortunately, however, the results cannot explain the JPH203 resistance profile.

Given that compensatory leucine supply is unlikely to occur following JPH203 treatment, the second possibility was tested. However, blockade of NF-kB, Akt, MAPK, p38, JNK signals did not augment JPH203 efficacy, suggesting that these survival signaling pathways are not involved in the JPH203 resistance of TE cells.

Taken together, the mechanisms underlying JPH203 resistance in TE cells are currently unknown. Our results indicate that JPH203 resistance can be acquired not always by lack of LAT 1 but also by other unidentified factors. Identification of such resistance factors will be essential for facilitating precision medicine in JPH203 treatment. Furthermore, identification of the resistance factors will pave the way for the development of an approach to overcome resistance, which will dramatically improve the efficacy of JPH203-based therapy.

Considering their clinical importance, we should keep striving to identify JPH203 resistance factor(s) in the future. For example, comparison of trans-omics profiling between JPH203-sensitive and -insensitive cells may provide some clues on new molecules and intracellular pathways involved in the resistance to JPH203. Moreover, the possibility that JPH203 has an additional pharmacological target other than LAT1 should be borne in mind. If intracellular JPH203 plays a certain role in its anticancer activity, it is worthwhile to consider the potential involvement of intracellular JPH203 metabolism and excretion.

To summarize, our results clearly show that JPH203 effectively inhibited the growth of gastric and colon cancer cells, paving the way for further development of JPH203. Also, LAT1 was not an exclusive factor predicting JPH203 efficacy. The primary factors causing JPH203 resistance remain elusive. However, since biomarkers for efficacy prediction will play an important role in JPH203-based cancer treatments, efforts for their identification should be made in the future.

\section{Acknowledgements}

The Authors would like to thank Dr. Tetsuro Nishihira (Tohoku University, Sendai, Japan) for providing TE cell series and Ms. Keiko Iida for her technical assistance. This work was supported in part by grants from the Japan Society for the Promotion of Science (JSPS KAKENHI 26461258 and 18K08200 [N.A.]), Strategic Research Foundation Grant-aided Project for Private Universities (S1412001), Gout Research Foundation of Japan, and Cancer Research Funds for Patients and Family by Medical and Welfare Network Chiba (N.A.).

\section{References}

1 McGivan JD and Pastor-Anglada M: Regulatory and molecular aspects of mammalian amino acid transport. Biochem J 299: 321-334, 1994.

2 Christensen HN: Role of amino acid transport and countertransport in nutrition and metabolism. Physiol Rev 70: 43-77, 1990.

3 Morimoto E, Kanai Y, Kim DK, Chairoungdua A, Choi HW, Wempe MF, Anzai N and Endou H: Establishment and characterization of mammalian cell lines stably expressing human L-type amino acid transporters. J Pharmacol Sci 108: 505-516, 2008.

4 Sakata T, Ferdous G, Tsuruta T, Satoh T, Baba S, Muto T, Ueno A, Kanai Y, Endou $\mathrm{H}$ and Okayasu I: L-type amino acid transporter 1 as a novel biomarker for high-grade malignancy in prostate cancer. Pathol Int 59: 7-18, 2009.

5 Furuya M, Horiguchi J, Nakajima H, Kanai Y and Oyama T: Correlation of L-type amino acid transporter 1 and CD98 expression with triple negative breast cancer prognosis. Cancer Sci 103: 382-389, 2012.

6 Kaira K, Oriuchi N, Imai H, Shimizu K, Yanagitani N, Sunaga N, Hisada T, Kawashima O, Iijima H, Ishizuka T, Kanai Y, Endou H, Nakajima T and Mori M: Expression of L-type amino acid transporter 1 (LAT1) in neuroendocrine tumors of the lung. Pathol Res Pract 204: 553-561, 2008.

7 Kaira K, Sunose Y, Arakawa K, Ogawa T, Sunaga N, Shimizu $\mathrm{K}$, Tominaga $\mathrm{H}$, Oriuchi $\mathrm{N}$, Itoh $\mathrm{H}$, Nagamori S, Kanai $\mathrm{Y}$, Segawa A, Furuya M, Mori M, Oyama T and Takeyoshi I: Prognostic significance of L-type amino-acid transporter 1 expression in surgically resected pancreatic cancer. Br J Cancer 107: 632-638, 2012.

8 Kobayashi H, Ishii Y and Takayama T: Expression of L-type amino acid transporter 1 (LAT1) in esophageal carcinoma. J Surg Oncol 90: 233-238, 2005.

9 Honjo H, Kaira K, Miyazaki T, Yokobori T, Kanai Y, Nagamori $\mathrm{S}$, Oyama T, Asao $\mathrm{T}$ and Kuwano $\mathrm{H}$ : Clinicopathological significance of LAT1 and ASCT2 in patients with surgically resected esophageal squamous cell carcinoma. J Surg Oncol 113: 381-389, 2016.

10 Ichinoe M, Mikami T, Yoshida T, Igawa I, Tsuruta T, Nakada N, Anzai N, Suzuki Y, Endou H and Okayasu I: High expression of L-type amino-acid transporter 1 (LAT1) in gastric carcinomas: comparison with non-cancerous lesions: Pathol Int 61: 281-289, 2011.

11 Hayase S, Kumamoto K, Saito K, Kofunato Y, Sato Y, Okayama $\mathrm{H}$, Miyamoto K, Ohki S and Takenoshita S: L-type amino acid transporter 1 expression is upregulated and associated with cellular proliferation in colorectal cancer. Oncol Lett 14: 74107416, 2017. 
12 Oda K, Hosoda N, Endo H, Saito K, Tsujihara K, Yamamura M, Sakata T, Anzai N, Wempe MF, Kanai Y and Endou H: L-type amino acid transporter 1 inhibitors inhibit tumor cell growth. Cancer Sci 101: 173-179, 2010.

13 Wempe MF, Rice PJ, Lightner JW, Jutabha P, Hayashi M, Anzai $\mathrm{N}$, Wakui S, Kusuhara $\mathrm{H}$, Sugiyama $\mathrm{Y}$ and Endou $\mathrm{H}$ : Metabolism and pharmacokinetic studies of JPH203, an L-amino acid transporter 1 (LAT1) selective compound. Drug Metab Pharmacokinet 27: 155-161, 2012.

14 Yothaisong S, Dokduang H, Anzai N, Hayashi K, Namwat N, Yongvanit $\mathrm{P}$, Sangkhamanon S, Jutabha $\mathrm{P}$, Endou H and Loilome W: Inhibition of L-type amino acid transporter 1 activity as a new therapeutic target for cholangiocarcinoma treatment. Tumor Biol 39: 1-14, 2017.

15 Yun DW, Lee SA, Park MG, Kim JS, Yu SK, Park MR, Kim SG, Oh JS, Kim CS, Kim HJ, Kim JS, Chun HS, Kanai Y, Endou H, Wempe MF and Kim DK: JPH203, an L-type amino acid transporter 1-selective compound, induces apoptosis of YD-38 human oral cancer cells. J Pharmacol Sci 124: 208-217, 2014.

16 Choi DW, Kim DK, Kanai Y, Wempe MF, Endou H and Kim JK: JPH203, a selective L-type amino acid transporter 1 inhibitor, induces mitochondria-dependent apoptosis in Saos2 human osteosarcoma cells. Korean J Physiol Pharmacol 21: 599-607, 2017.
17 Hayashi K, Jutabha P, Maeda S, Supak Y, Ouchi M, Endou H, Fujita T, Chida M and Anzai N: LAT1 acts as a crucial transporter of amino acids in human thymic carcinoma cells. J Pharmacol Sci 132: 201-204, 2016.

18 Okano N, Kawai K, Yamauchi Y, Kobayashi T, Naruge D, Nagashima F, Endou $\mathrm{H}$ and Furuse J: First-in-human phase I study of JPH203 in patients with advanced solid tumors. J Clin Oncol 36: 419, 2018.

$19 \mathrm{He} \mathrm{C}$ and Klionsky DJ: Regulation mechanisms and signaling pathways of autophagy. Annu Rev Genet 43: 67-93, 2009.

20 Nakatogawa H, Suzuki K, Kamada Y and Ohsumi Y: Dynamics and diversity in autophagy mechanisms: lessons from yeast. Nat Rev Mol Cell Biol 10: 458-467, 2009.

Received November 14, 2018

Revised November 30, 2018

Accepted December 3, 2018 